\title{
Kompetenzen für Spezialistinnen und Spezialisten in Palliative Care
}

\author{
Der von der Arbeitsgruppe SwissEduc im Auftrag von palliative ch erarbeitete \\ Kompetenzenkatalog liegt nach rund dreijähriger Arbeit vor. Im hundertseitigen \\ Dokument werden die Kompetenzen für die Berufsgruppen des medizinisch-pflege- \\ rischen und des psycho-sozialen Bereiches auf Ausbildungs-Niveau B2 detailliert \\ beschrieben.
}

Eva Waldmann, Daniel Büche

Im Auftrag der Arbeitsgruppe SwissEduc
Korrespondenz:

Dr. med Daniel Büche, M. Sc. Leiter Palliativzentrum Kantonsspital St.Gallen CH-9007 St. Gallen daniel.bueche[at]kssg.ch
Palliative Care gewinnt in ganz Europa an Bedeutung, eine einheitliche Anerkennung des Fachgebietes fehlt allerdings weitgehend. Gerade deshalb kommt der Beschreibung von Kompetenzen für Spezialistinnen und Spezialisten in Palliative Care eine zentrale Bedeutung zu. Der jetzt vorliegende Kompetenzenkatalog bildet benötigtes Wissen, Fähigkeiten und Handlungsweisen systematisch ab und erleichtert es Bildungsanbietern, Lerninhalte für Nachdiplomstudiengänge zusammenzustellen. Im Rahmen der interprofessionellen Zusammenarbeit dient er der Abgrenzung und Festlegung berufsspezifischer Eigenarten. Im Bereich des Qualitätsmanagements lassen sich Arbeitsstandards für einzelne Berufe nun genauer definieren. Fachpersonen können das Dokument für eine persönliche Standortbestimmung nutzen oder ein Lernportfolio erstellen. loping a Curriculum) gewählt. Sie orientiert sich nicht an Idealen, sondern widerspiegelt den beruflichen Alltag der Spezialisten. Unter Anleitung eines geschulten Moderators analysierte und definierte eine Gruppe von ausgewählten Berufsleuten das eigene Fachgebiet.

\section{Ausblick}

Der Katalog steht nun auf der Website von palliative ch als Download zur Verfügung. Für die Ärzte ist aktuell das Niveau B2 (Spezialisten in Palliative Care) beschrieben. Die Fachärztegruppe der nationalen Gesellschaft für Palliative Care ist daran, mit allen Vertretern der Facharzttitel die für den jeweiligen FMH-Titel (WBO) relevanten Palliativ-Kompetenz zu definieren. Dies im Auftrag und in enger Zusammenarbeit des SIWF. Ebenfalls wird ein Fähigkeitsausweis

\section{Der Katalog will Ansporn sein, sich seiner Fachkompetenzen bewusst zu werden und diese laufend weiterzuentwickeln.}

\section{Kompetenz als Gesamtheit potentieller Verhaltensweisen}

Die Betreuung eines Palliativpatienten setzt Wissen um komplexe Zusammenhänge voraus. Laut dem Pädagogen Philippe Meirieu werden Kompetenzen verstanden als die Gesamtheit potentieller Verhaltensweisen (affektiv, kognitiv und psychomotorisch), dank derer ein Individuum komplexe Tätigkeiten effizient ausüben kann. Kompetenzen sind vielschichtig und dynamisch und in diesem Sinne bestens geeignet, die facettenreichen, interdisziplinären Aufgaben der Palliative Care zu beschreiben.

Um ein möglichst praxisnahes Bild der Palliative Care in der Schweiz zu zeichnen, wurde für die Erarbeitung des Kataloges die DACUM-Methode (Deve- für Palliativmedizin diskutiert. Auch hier besteht eine Zusammenarbeit mit dem SIWF und dem BAG. Der Katalog zeigt deutlich, dass Palliative Care in der Schweiz eine noch junge Disziplin ist. So gibt es Kompetenzbereiche, in denen noch keine oder sehr wenige allgemeine Kompetenzen benannt sind. Das Selbstverständnis der Berufsleute in Palliative Care braucht Zeit, um weiter zu wachsen und sich auszudifferenzieren. In diesem Sinne soll der vorliegende Katalog Ansporn sein, sich der Fachkompetenzen bewusst zu werden und diese laufend weiterzuentwickeln.

Weitere Informationen unter www.palliative.ch Rubrik Fachportal, Standards, Aus-, Weiter- und Fortbildung. 